LETTER TO THE EDITOR

\title{
Phase behavior of hard spheres confined between parallel hard plates: Manipulation of colloidal crystal structures by confinement
}

\author{
Andrea Fortini and Marjolein Dijkstra \\ Soft Condensed Matter, Utrecht University, Princetonplein 5, 3584 CC Utrecht, \\ The Netherlands. \\ E-mail: a.fortini@phys.uu.nl
}

\begin{abstract}
We study the phase behavior of hard spheres confined between two parallel hard plates using extensive computer simulations. We determine the full equilibrium phase diagram for arbitrary densities and plate separations from one to five hard-sphere diameters using free energy calculations. We find a first-order fluid-solid transition, which corresponds to either capillary freezing or melting depending on the plate separation. The coexisting solid phase consists of crystalline layers with either triangular $(\triangle)$ or square $(\square)$ symmetry. Increasing the plate separation, we find a sequence of crystal structures from $\cdots n \triangle \rightarrow(n+1) \square \rightarrow(n+1) \triangle \cdots$, where $n$ is the number of crystal layers, in agreement with experiments on colloids. At high densities, the transition between square to triangular phases are intervened by intermediate structures, e.g., prism, buckled, and rhombic phases.
\end{abstract}




\section{Introduction}

The physics of confined systems is important in different fields of modern technology, like lubrication, adhesion and nanotechnology. The study of simple models is instrumental in understanding the behavior of complex systems. As such the hardsphere system plays an important role in statistical physics; it serves as a reference system for determining the structure and phase behavior of complex fluids, both in theory and simulations. The bulk phase behavior of hard spheres is now well understood. At sufficiently high densities, the spheres can maximize their entropy by forming an ordered crystal phase [1, 2]. The insertion of a hard wall in such a fluid decreases the number of hard-sphere configurations. The system can increase its entropy by the spontaneous formation of crystalline layers with triangular symmetry, the (111) plane, at the wall, while the bulk is still a fluid [3]. This effect is known as prefreezing, and is analogous to complete wetting by fluids at solid substrates. It is induced by the presence of a single wall and should not be confused with capillary freezing. Capillary freezing denotes the phenomenon of confinement induced freezing of the whole fluid in the pore at thermodynamic state points where the bulk is still a fluid. This transition depends strongly on the plate separation. The opposite phenomenon, called capillary melting, can also occur. The capillary induces melting for thermodynamic state points that correspond to a crystal in the bulk. Confinement can also change dramatically the equilibrium crystal structure. In 1983 Pieranski [4] reported a sequence of layered solid structures with triangular and square symmetry for colloidal hard spheres confined in a wedge. The sequence of high density structures is determined more accurately in recent experiments [5, 6], reporting the observation of prism phases with both square and triangular symmetry. Recently Cohen [7] studied configurations of confined hard spheres under shear, demonstrating the importance of the equilibrium configurations in the rheological properties. Despite the great number of theoretical and simulation studies on confined hard spheres 8, 9, 10], the full equilibrium phase behavior is yet unknown. In fact, many of the previous studies were based on an order parameter analysis, which fails dramatically in discriminating the different structures at high densities and large plate separations. More importantly, free energy calculations of confined hard spheres are prohibited so far due to the lack of an efficient thermodynamic integration path which relates the free energy of interest to that of a reference system, while a further complication arises from the enormous number of possible solid phases that has to be considered. Hence, it is unresolved whether the experimentally observed phases are stabilized kinetically or are thermodynamically stable.

In this letter, we present a novel efficient thermodynamic integration path that enable us to calculate the free energy of densely packed and confined hard spheres, with high accuracy close to the fluid-solid transition. This method allow us to determine for the first time the stability of the structures found in experiments. To this end we perform explicit free energy calculations to map out the full phase diagram for plate separations from 1 to 5 hard-sphere diameters. We report a dazzling number of thermodynamically stable crystal structures (26!) including triangular, square, buckling, rhombic, and prism phases, and a cascade of corresponding solidsolid transformations. In addition, the free energy calculations allow us to determine the chemical potential at coexistence, that was unaccessible in previous simulations. From the analysis of the chemical potential, we find an intriguing sequence of capillary freezing and melting transitions coupled to a structural phase transition of the confined 


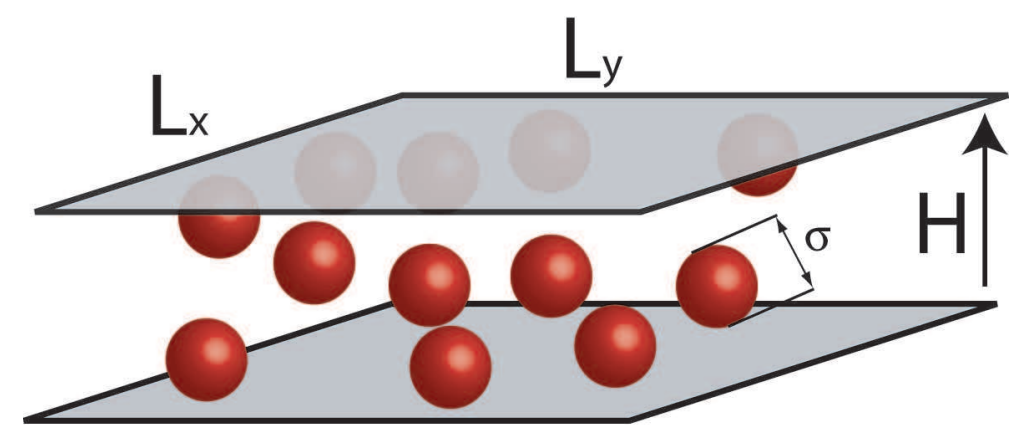

Figure 1. (Color online) Illustration of hard spheres with diameter $\sigma$, confined between parallel hard plates of area $A=L_{x} L_{y}$ and a separation distance $H$.

crystal. We note that our new method and results are also relevant for confined simple fluids [12, 11, 13] and self-assembled biological systems [14]. In addition, the structure of dense packings of spheres explains the shape of for instance snowflakes, bee honeycombs, and foams, and it is of great importance for fundamental research, e.g., solid state physics and crystallography, and for applications like communication science or powder technology [15].

\section{Model and Method}

Our model system consists of $N$ hard spheres with diameter $\sigma$, confined between two parallel hard plates of area $A=L_{x} L_{y}$ (Fig. 11). In each layer we used approximately 200 particles. We use the packing fraction $\eta=\pi \sigma^{3} N /(6 A H)$ as a dimensionless density, where $H$ is the distance between two plates. We determine the equilibrium phase diagram by performing Monte Carlo (MC) simulations in a box, which is allowed to change its shape to accommodate different types of crystals; the ratio $L_{x} / L_{y}$ may vary while $H$ and $A$ are fixed. Trial solid structures are obtained from crystals with triangular or square symmetry relaxed with MC moves while slowly increasing the density by expanding the spheres. The free energy $F$ for the resulting equilibrated structures is calculated as a function of $\eta$ and $H$. We use the standard thermodynamic integration technique 16, 17, but with a new and efficient path based on penetrable potentials, that enable us to change gradually from a non-interacting system to the confined hard-core system of interest. The sphere-sphere potential reads

$$
v_{i j}\left(R_{i j}\right)=\left\{\begin{array}{ll}
\epsilon \exp \left(-\mathrm{A} R_{i j}\right) & \text { if } R_{i j}<\sigma_{c} \\
0 & \text { otherwise }
\end{array},\right.
$$

and the wall-fluid potential

$$
v_{w i}(z)=\left\{\begin{array}{ll}
\epsilon \exp \left(-\mathrm{B} z_{i}\right) & \text { if } z_{i}<\sigma_{c} / 2 \\
0 & \text { otherwise }
\end{array},\right.
$$

where $R_{i j}$ is the distance between spheres $i$ and $j, z_{i}$ is the distance of sphere $i$ to the nearest wall, $\mathrm{A}$ and $\mathrm{B}$ are adjustable parameters that are kept fixed during the 


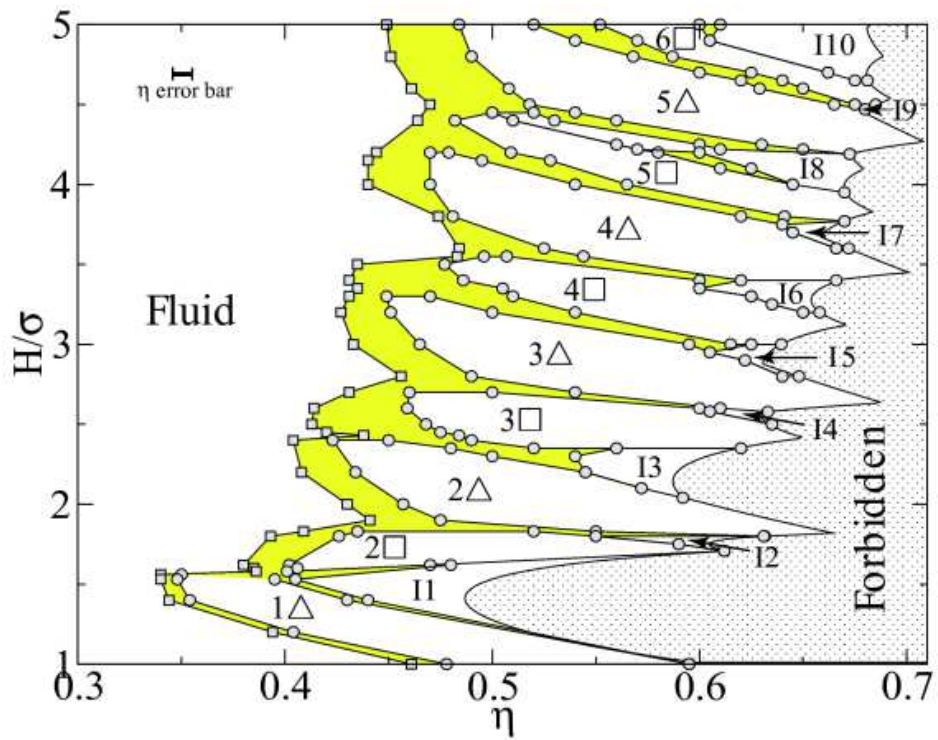

Figure 2. (Color online) The equilibrium phase diagram of hard spheres with diameter $\sigma$ confined between two parallel hard walls in the plate separation $H$ packing fraction $\eta$ representation. The white, yellow and dotted regions indicate the stable one-phase region, the two-phase coexistence region, and the forbidden region, respectively.

Table 1. List of intermediate structures In as found in our simulations and in the experiments of Fontecha 6 .

\begin{tabular}{llll}
\hline Phase & Transition & Simulation $\ddagger$ & Experiment \\
\hline I1 & $1 \triangle \rightarrow 2 \square$ & $2 \mathcal{B}$ & $2 \mathcal{B}$ \\
I2 & $2 \square \rightarrow 2 \triangle$ & $2 \mathcal{R}$ & $2 \mathcal{R}$ \\
I3 & $2 \triangle \rightarrow 3 \square$ & $2 \mathcal{P}_{\triangle}+2 \mathcal{P}_{\square}$ & $2 \mathcal{P}_{\triangle}+2 \mathcal{P}_{\square}$ \\
I4 & $3 \square \rightarrow 3 \triangle$ & $3 \mathcal{R}+3 \mathcal{P}_{\square}+(3 \mathcal{B})$ & $3 \mathcal{R}+3 \mathcal{P}_{\square}+3 \mathcal{P}_{\triangle}$ \\
I5 & $3 \triangle \rightarrow 4 \square$ & $3 \mathcal{P}_{\triangle}+4 \mathcal{B}$ & $3 \mathcal{P}_{\square}$ \\
I6 & $4 \square \rightarrow 4 \triangle$ & $4 \mathcal{P}_{\square}+4 \mathcal{R}+4 \mathcal{P}_{\triangle}$ & $4 \mathcal{P}_{\square}+4 \mathcal{P}_{\triangle}$ \\
I7 & $4 \triangle \rightarrow 5 \square$ & $4 \mathcal{P}_{\triangle}$ & $4 \mathcal{P}_{\triangle}, 4 \mathcal{P}_{\square}, H \S$ \\
I8 & $5 \square \rightarrow 5 \triangle$ & $5 \mathcal{P}_{\square}+4 \mathcal{P}_{\triangle}+\left(5 \mathcal{P}_{\triangle}\right)+5 \mathcal{R}$ & $5 \mathcal{P} \|$ \\
I9 & $5 \triangle \rightarrow 6 \square$ & $5 \mathcal{P}_{\triangle}$ & no data \\
I10 & $6 \square \rightarrow 6 \triangle$ & $5 \mathcal{P}_{\square}+5 \mathcal{P}_{\triangle}$ & no data \\
\hline
\end{tabular}

simulations, and $\epsilon$ is the integration parameter. The limit $\epsilon \rightarrow \infty$ yields the hard-core interaction, but convergence of the thermodynamic integration is already obtained for $\epsilon \sim 70 k_{B} T$. The reference states $\left(\epsilon=0 k_{B} T\right)$ are the ideal gas and the Einstein crystal for the fluid and solid phase, respectively. We use a 21-point Gaussian quadrature for the numerical integrations and the ensemble averages are calculated from runs with $40000 \mathrm{MC}$ cycles (attempts to displace each particle once), after first equilibrating the system during $20000 \mathrm{MC}$ cycles. We determine phase coexistence by equating the grand potentials $\Omega=F-\mu N$ [18]. 


\section{Results}

To validate this approach, we perform simulations of a bulk system of hard spheres and we find that the packing fractions of the coexisting fluid and face-centered-cubic (fcc) solid phase are given by $\eta_{f}=0.4915 \pm 0.0005$ and $\eta_{s}=0.5428 \pm 0.0005$, respectively. The pressure and the chemical potential at coexistence are $\beta P \sigma^{3}=11.57 \pm 0.10$ and $\beta \mu=16.08 \pm 0.10$. These results are in good agreement with earlier results [1] [19]. Furthermore, to validate the approach for confined systems, we determine at bulk coexistence the wall-fluid interfacial tension $\beta \gamma_{\mathrm{wf}} \sigma^{2}=1.990 \pm 0.007$, and the wall-solid interfacial tension for the (111) and (100) planes of the fcc phase, $\beta \gamma_{\mathrm{ws}}^{111} \sigma^{2}=1.457 \pm 0.018$ and $\beta \gamma_{\mathrm{ws}}^{100} \sigma^{2}=2.106 \pm 0.021$. Our results are in agreement with previous simulations [20, but the statistical error is one order of magnitude smaller due to our new thermodynamic integration path.

Employing this approach we determine the phase behavior of confined hard spheres for plate separations $1<H / \sigma \leq 5$. Fig. 2 displays the full phase diagram based on free energy calculations in the $H-\eta$ representation. The white regions of the phase diagram denote the stable one-phase regions. The (yellow) shaded regions indicate coexistence between fluid and solid or two solid phases, and the dotted region is forbidden as it exceeds the maximum packing fraction of confined hard spheres. At low densities, we observe a stable fluid phase followed by a fluid-solid transition upon increasing the density. The oscillations in the freezing and melting lines reflect the (in)commensurability of the crystal structures with the available space between the walls. For the crystal phases, we follow the convention introduced by Pieranski [4, where $n \triangle$ denotes a stack of $n$ triangular layers, and $n \square$ a stack of $n$ square layers. For $H / \sigma \rightarrow 1$, the stable crystal phase consists of a single triangular layer $1 \triangle$, which packs more efficiently than the square layer. As the gap between the plates increases, crystal slabs with triangular (Fig. 3(a)) and square packings (Fig. 3(b)) are alternately stable. We find the characteristic sequence $\cdots n \triangle \rightarrow(n+1) \square \rightarrow(n+1) \triangle$, which consists of an $n \triangle \rightarrow(n+1) \square$ transformation where both the number of layers and the symmetry change followed by an $(n+1) \square \rightarrow(n+1) \triangle$ transformation where only the symmetry changes. This sequence is driven by a competition of a smaller height of $n$ square layers compared to $n$ triangular layers and a more efficient packing of triangular layers w.r.t. square layers. When the available gap is larger than required for the $n \triangle$ structure, but smaller than for $(n+1) \square$, intermediate structures may become stable. Similar arguments can be used for the intervention of intermediate structures in the $(n+1) \square \rightarrow(n+1) \triangle$ transformation. Especially at high packing fractions, the spheres can increase their packing by adopting interpolating structures. In Fig. 2 we report the boundaries of the interpolating regions In. Each region represents one or more interpolating structures, that are listed in Tab. 1 according to the standard notation. Within the resolution of our simulations, it is difficult to draw the phase boundaries of all the intermediate structures in In, but in Tab. 1 the thermodynamically stable structures are listed in the order they appear upon increasing $H$ and $\eta$. We also compare our sequence of structures with the experimental one [6]. The experiments considered charged particles, but we do not expect that the soft repulsion has a strong effect on the observed structures at high densities. The agreement is excellent at small plate separations. The buckling phase $2 \mathcal{B}$ (Fig. B(c)) interpolates between $1 \triangle$ and $2 \square$. In the $2 \mathcal{B}$, the $1 \triangle$ is split into two sublayers consisting of rows that are displaced in height and which can transform smoothly into $2 \square$ upon increasing the gap. The rhombic phase $2 \mathcal{R}$ (Fig. [3(d)) is found between 
$2 \square$ and $2 \triangle$. The rhombic phase is also stable between $n \square$ and $n \triangle$ for $n \leq 5$, but not in the whole region. In addition, we find that at higher $n$ the interpolating structures are mainly prism phases. In agreement with experiments, we find two types of prisms, one with a square base $n \mathcal{P}_{\square}$ (Fig. B(e)), and one with a triangular base $n \mathcal{P}_{\triangle}$ (Fig. B](f)), where $n$ indicates the number of particles in the prism base. As shown in Fig. B](g), (h), these structures display large gaps as a result of periodically repeated stacking faults in the packing which, nevertheless, allow particles to pack more efficiently, than a phase consisting of parallel planes of particles. For $n>3$, differences between simulations and experiments emerge. We find that the stability region of interpolating structures between $n \triangle$, and $(n+1) \square$ decreases for larger $H$, becoming invisible on the scale of Fig. 2 for $I 9=5 \triangle \rightarrow 6 \square$. On the other hand, the region of stability of the interpolating structures between $n \square$ and $n \triangle$ increases while increasing the wall separation, becoming stable also at low packing fractions for the transitions $I 8=5 \square \rightarrow 5 \triangle$, and possibly $I 10=6 \square \rightarrow 6 \triangle$. We also note that the solid-solid transitions are first-order with a clear density jump at low $\eta$, but they get weaker (and maybe even continuous) upon approaching the maximum packing limit. In addition, the rhombic and buckling phases are highly degenerate as we find zig-zag and linear buckling or rhombic phases, and a combination of those.

We now turn our attention to the fluid-solid transition. In Fig. 4 we plot the chemical potential $\beta \mu^{\text {cap }}$ at the freezing transition of the confined system as a function of $H$. The freezing for crystal slabs with a triangular symmetry are denoted by triangles, while the square symmetry is displayed by squares. We find strong oscillations in the chemical potential reminiscent to the (in)commensurability of the crystal structures with plate separation. The highest values for $\beta \mu^{\text {cap }}$ are reached at the transition region $n \triangle \rightarrow(n+1) \square$, corresponding to plate separations where both structures are incommensurate and hence unfavorable. In this regime, $\beta \mu^{\text {cap }}$ can reach values that are higher than the bulk freezing chemical potential $\beta \mu^{\text {bulk }}$ (the black vertical line in Fig. (4), corresponding to capillary melting, while the freezing transitions with $\beta \mu^{\text {cap }}$ lower than the bulk value correspond to capillary freezing. Hence, we find a reentrant capillary freezing/melting behavior for wall separations $1<H / \sigma<3.5$. In addition, we compare our results with the predictions of the Kelvin equation [21]: $\beta \mu^{\text {cap }}=\beta \mu^{\text {bulk }}-\pi \sigma^{3} / 3 H\left(\gamma_{w f}-\gamma_{w s}\right) /\left(\eta_{s}-\eta_{f}\right)$ using the parameters determined in our simulations. The thick dashed line in Fig. 4 is the prediction of the Kelvin equation for the (111) crystal plane (triangular order) at the walls, while the dotted line is that for the (100) plane (square order). The Kelvin equation predicts capillary freezing for the triangular structure and capillary melting for the square structures. The Kelvin equation predictions are in reasonable agreement with our simulations for triangular order for wall separation as small as $H / \sigma \sim 4$, but deviates for smaller $H$, while the prediction for the square structure is in agreement only at very small $H$. It is surprising to find qualitative agreement at small $H$ since the Kelvin equation is valid in the limit $H / \sigma \rightarrow \infty$.

\section{Conclusion}

In summary, we have calculated the equilibrium phase diagram of confined hard spheres using free energy calculations with a novel integration path. The high density sequence of structures is in good agreement with experimental results. We find that the prism phases are thermodynamically stable also at lower densities, and this work will, hopefully, stimulate further experimental investigations, for a quantitative comparison 
(a)

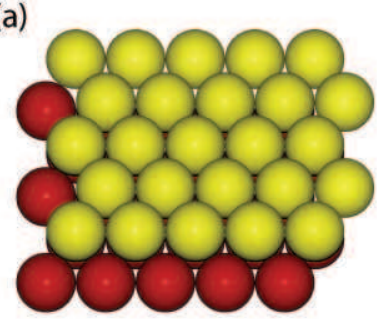

(c)

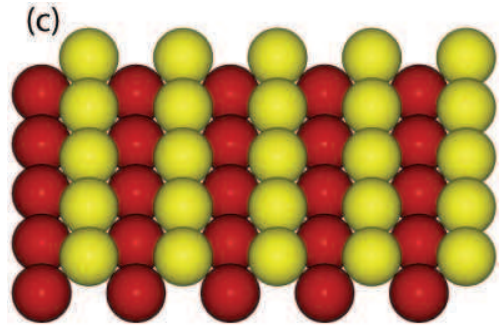

(e)

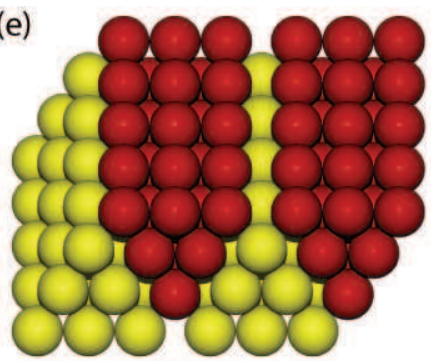

(g) (b)

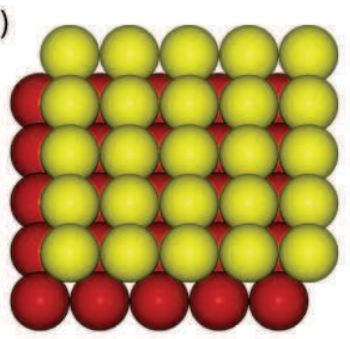

(d)

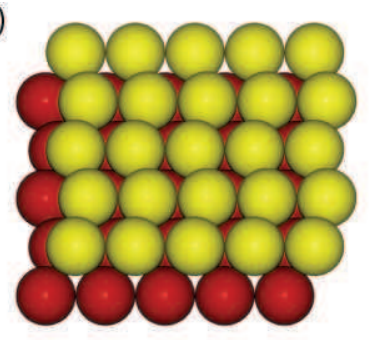

(f)

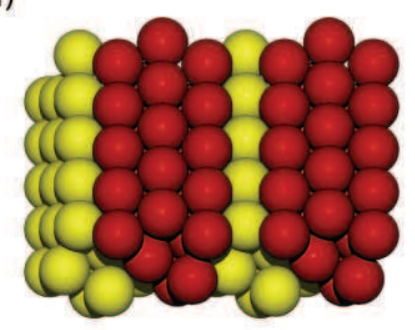

(h)

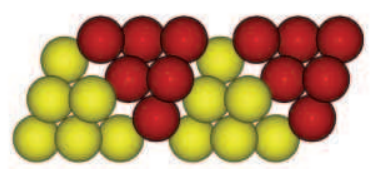

Figure 3. (Color online) Stable solid structures of confined hard spheres. (a) The triangular phase $2 \triangle$ (b) The square phase $2 \square$ (c) The buckling phase $2 \mathcal{B}$ (d) The rhombic phase $2 \mathcal{R}$ (e),(g) The prism phase with square symmetry $3 \mathcal{P}_{\square}$ (f),(h) The prism phase with triangular symmetry $3 \mathcal{P}_{\triangle}$. In (a)-(f) the point of view is at an angle of $30^{\circ}$ to the $\mathrm{z}$ direction. In $(\mathrm{g}),(\mathrm{h})$ the point of view is at an angle of $90^{\circ}$. Different shades (colors) indicate particles in different planes $((\mathrm{a})-(\mathrm{d}))$ or particles belonging to different prism structures $((\mathrm{e})-(\mathrm{h}))$. 


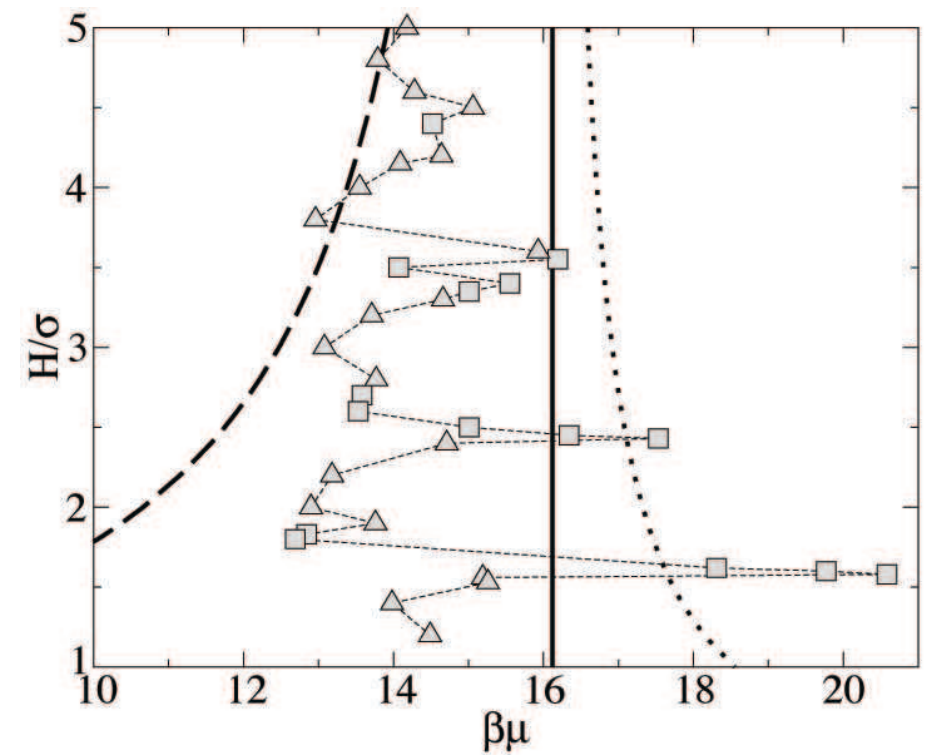

Figure 4. Chemical potential $\beta \mu$ at fluid-solid coexistence, for different wall separations $H / \sigma$. The symbols are the simulation results for the triangular $(\triangle)$ and square structures $(\square)$. The thin dashed line is a guide to the eye. The thick continuous line indicate the value of the bulk freezing chemical potential $\beta \mu=16.08$. The thick dashed and dotted curves are the prediction of the Kelvin equation for the 111, and 100 planes parallel to the walls, respectively.

at intermediate packing fractions. In addition, our results show an intriguing sequence of melting and freezing transitions upon increasing the distance between the walls of a slit which is in contact with a bulk reservoir. The mechanical behavior is therefore very sensitive on the degree of confinement, and the knowledge of the phase diagram can help the understanding and fabrication of new materials. The transition from confined to bulk behavior, and the interface between different solid structures (studied in lower dimensions in Ref. [22]) represent interesting directions for future investigations.

\section{Acknowledgments}

We thank M. Schmidt for inspiring discussions. This work is part of the research program of the Stichting voor Fundamenteel Onderzoek der Materie (FOM), that is financially supported by the Nederlandse Organisatie voor Wetenschappelijk Onderzoek (NWO). We thank the Dutch National Computer Facilities foundation for granting access to TERAS and ASTER supercomputers.

\section{References}

[1] W.G. Hoover and F.M. Ree, J. Chem. Phys. 49, 3609 (1968).

[2] P. N. Pusey and W. van Megen, Nature (London) 320, 340 (1986).

[3] M. Dijkstra, Phys. Rev. Lett. 93, 108303 (2004); D.J. Courtemanche and F. van Swol, Phys. Rev. Lett. 69, 2078 (1992).

[4] P. Pieranski and L. Strzelecki and B. Pansu, Phys. Rev. Lett. 50, 900 (1983).

[5] S. Neser et al., Phys. Rev. Lett. 79, 2348 (1997). 
[6] A.B. Fontecha et al., J. Phys.: Cond. Matt. 17, S2779 (2005).

[7] I. Cohen et al., Phys. Rev. Lett. 93, (2004).

[8] M. Schmidt and H. Löwen, Phys. Rev. Lett. 76, 4552 (1996); Phys. Rev. E 55, 7228 (1997).

[9] R. Zangi and S.A. Rice, Phys. Rev. E 61, 660 (2000); ibid 61, 671 (2000).

[10] R. Messina and H. Löwen, Phys. Rev. Lett. 91, 146101 (2003); Phys. Rev. E 73, 011405 (2006).

[11] J. Gao et al., Phys. Rev. Lett. 79, 705 (1997).

[12] J. Klein and E. Kumacheva, Science. 269, 816 (1995).

[13] C. Ghatak and K. G. Ayappa, Phys. Rev. E. 64, 051507 (2001).

[14] A. Ciach, Progr. Colloid Polym. Sci. 129, 40 (2004).

[15] J.H. Conway and N.J.A. Sloane, Sphere Packings, Lattices and Groups (Springer, New York, 1993); T. Aste and D. Weaire, The Pursuit of Perfect Packing (IOP, Bristol, 2000).

[16] D. Frenkel and B. Smit, Understanding Molecular Simulation 2nd edition (Academic Press, 2002).

[17] A. Fortini et al., Phys. Rev. E 71, 051403 (2005).

[18] R. Evans and U. Marini Bettolo Marconi, J. Chem. Phys. 86, 7138 (1987).

[19] R.L. Davidchack and B.B. Laird, J. Chem. Phys. 108, 9452 (1998).

[20] M. Heni and H. Löwen, Phys. Rev. E 60, 7057 (1999).

[21] J.S. Rowlinson and B. Widom, Molecular Theory of Capillarity (Dover, New York, 2002).

[22] D. Chaudhuri and S. Sengupta. Phys. Rev. Lett. 93, 115702 ( 2004). 